Pacific Journal of Mathematic 


\title{
MODULUS OF A BOUNDARY COMPONENT
}

\author{
Martin JURChESCU
}

\section{§1. Preliminaries and Summary}

1.1 Preliminary definitions. Let $R$ be an open Riemann surface, and let $\left\{G_{n}\right\}(n=1,2, \cdots)$ be an infinite sequence of subregions of $R$ such that:

(a) the relative boundary of each $G_{n}$ is compact,

(b) $G_{n} \supset G_{n+1}$, and

(c) $\bigcap_{n=1}^{\infty} \bar{G}_{n}=0$.

$\left\{G_{n}\right\}^{n=1}$ is said to define a boundary component $\gamma$ of $R$ in the sense of Kerékjártó [6] and Stoilow [16]. Here two sequences of subregions $\left\{G_{n}\right\}$ and $\left\{G_{n}^{\prime}\right\}$ are considered to be equivalent and to define the same $r$ if each region $G_{n}$ includes a region $G_{m}^{\prime}$. That this is a proper equivalence relation follows immediately.

Let $\gamma$ be a boundary component of $R$, and let $S$ be a subregion of $R$. If there exists a defining sequence $\left\{G_{n}\right\}$ of $\gamma$ with $G_{n_{0}}=S$, for some $n_{0}$, we call $S$ a neighborhood of $\gamma$. Throughout this paper we shall consider only neighborhoods $S$ of $\gamma$ such that the relative boundary of $S$ is a closed analytic Jordan curve $\gamma_{0}$.

By an exhaustion of $R$, we mean an infinite sequence $\left\{R_{n}\right\}(n=$ $1,2, \cdots)$ of subregions of $R$ as follows (see [16]):

(1) each $R_{n}$ is compact relative to $R$ and the relative boundary $\beta_{n}$ of $R_{n}$ consists of a finite number of closed analytic Jordan curves $\beta_{n i}$,

(2) $R_{n} \subset R_{n+1}$,

(3) $\bigcup R_{n}=R$, and

(4) each connected component $S_{n i}$ of $R-\bar{R}_{n}$ is non-compact (relative to $R$ ) and its boundary consists of a single curve $\beta_{n i}$.

Each set $R-\bar{R}_{n}$ is said to be a boundary neighborhood of $R$. It is easy to see that, for any boundary component $\gamma$ of $R$, there exists a single connected component $S_{n i}$ which is a neighborhood of $\gamma$.

A property is said to be a boundary property (respectively a $\gamma$-property) if the following is true. If a Riemann surface $R$ has the property then every Riemann surface $R^{\prime}$ which admits a conformal mapping from a boundary neighborhood of $R^{\prime}$ (a neighborhood of $\gamma^{\prime}$, where $\gamma^{\prime}$ is a boundary

Received April, 1, 1958, and in received form May 1, 1958. This paper is a part of the author's dissertation realized under the guidance of Professor Stoilow at Mathematical Institute of the R. P. P. Academy, Bucarest. The author wishes to express his gratitude to Professor Stoilow for his encouragement and permanent support. The author is also indebted to Dr. Kotaro Oikawa of Tokyo Institute of Technology who read the manuscript and made valuable remarks. 
component of $R^{\prime}$ ) onto a boundary neighborhood of $R$ (a neighborhood of $\gamma$ ) has the property.

Let $u$ be a harmonic function on a subregion $S$ of $R$. We shall denote by $\bar{u}$ the conjugate harmonic function of $u$ and by $D(u ; S)$ the Dirichlet integral of $u$ over $S$.

1.2. Capacity of a boundary component. Let $\gamma$ be a boundary component of an open Riemann surface $R, P_{0}$ a point of $R$, and $K_{z}:|z| \leqq 1$ a fixed parametric disc on $R$ with $z=0$ corresponding to $P_{0}$. Let $\left\{R_{n}\right\}$ be an exhaustion of $R$ with $P_{0} \in R_{1}$, and let $\gamma_{n}$ denote the curve $\beta_{n i}$ which separates $\gamma$ from $P_{0}$. This means that $\gamma_{n}$ separates a neighborhood of $\gamma$ from $P_{0}$.

We consider the class $\{t\}_{\gamma}$ of single-valued functions on $R$ which satisfy the following conditions:

(1.1) each $t$ is harmonic on $R-P_{0}$ and has the form

$$
t=\log |z|+h(z)
$$

in $K_{z}$, where $h$ is harmonic and $h(0)=0$.

$$
\int_{\gamma_{n}} d \bar{t}=2 \pi \text { and } \int_{\beta n i \neq \gamma n} d \bar{t}=0, \quad \text { for all } n,
$$

where $\gamma_{n}$ and $\beta_{n i}$ are described in the positive sense with respect to $R_{n}$.

We further consider the corresponding class $\{t\}_{\gamma_{n}}$ on $R_{n}$, and we denote by $t_{n}$ the function of this class with $t_{n}=k_{n}$ on $\gamma_{n}$ and $t_{n}=k_{n i}$ on $\beta_{n i} \neq \gamma_{n}$, where $k_{n}$ and $k_{n i}$ are real numbers.

The following theorem due to Sario is proved in [14] (see also Savage [15]). Let $t \in\{t\}_{\gamma}$, and let

$$
I(t)=\lim \frac{1}{2 \pi} \int_{\rho_{n}} t d \bar{t} .
$$

THEOREM 1. The sequence of functions $\left\{t_{n}\right\}$ is compact. Let $t_{\gamma}$ denote a limit function of $\left\{t_{n}\right\}$. Then we have the following conclusions:

$$
\begin{gathered}
t_{\gamma} \in\{t\}_{\gamma} \text { and, for any } t, \min I(t)=I\left(t_{\gamma}\right) . \\
I(t)=I\left(t_{\gamma}\right)+D\left(t-t_{\gamma} ; R\right) . \\
k_{n} \leqq k_{n+1} \text { and } I\left(t_{\gamma}\right)=\lim k_{n} \equiv k_{\gamma} .
\end{gathered}
$$

By (1.4), for $k_{\gamma}<\infty$, the minimizing function $t_{\gamma}$ is unique. $t_{\gamma}$ is called the capasity function of $R$ for $\gamma$, and the quantity $c_{\gamma}=e^{-k \gamma}$ is called the capasity of $\gamma$ (with respect to $K_{z}$ ). Let $z^{\prime}=a z+\cdots, a \neq 0$, be a new local parameter in the neighborhood of $P_{0}$, and let $c^{\prime}{ }_{\gamma}$ denote the capacity of $\gamma$ with respect to this local parameter. It follows, from the definition of the capacity, that 


$$
c_{\gamma}=|a| c_{\gamma}^{\prime} .
$$

Hence, the condition $c_{\gamma}=0$ is independent of the local parameter which is used in the neighborhood of $P_{0}$. Using Green's formula, it is easy to see that this condition is also independent of $P_{0}$. A boundary component $\gamma$ is called weak if it has a capacity $c_{\gamma}=0$. The class of Riemann surfaces for which all $\gamma$ are weak is denoted by $C_{\gamma}$. The boundary of a Riemann surface $R$ belonging to $C_{\gamma}$ is called absolutely disconnected $[14,15]$.

1.3. Summary. Let $R$ be an open Riemann surface, $r$ a boundary component of $R, S$ a neighborhood of $\gamma$, and $\gamma_{0}$ the relative boundary of $S$. The present paper deals with a conformal invariant of $S$ which is denoted by $\mu\left(S ; \gamma_{0}, \gamma\right)$ (or, simply, for fixed $S$, by $\mu_{\gamma}$ ) and is called the modulus of $S$ for $\gamma_{0}$ and $r$ (the modulus of $\gamma$ ).

In $\$ 2$ harmonic functions $u$ on $S$ with $u=0$ on $\gamma_{0}$ and satisfying conditions (2.3) are considered, and a theorem is proved which establishes the existence of a minimizing function $u_{\gamma}=u\left(z ; S ; \gamma_{0}, \gamma\right)$ for the Dirichlet integral $D(u ; S)$. The modulus is defined by setting $\mu_{\gamma}=D\left(u_{\gamma} ; S\right)$. The notion of a parabolic boundary component is defined by the condition $\mu_{\gamma}=\infty$, and a theorem is proved which shows the equivalence of parabolicity and weakness.

In $\S 3$ measurable conformal metrics are considered. An important minimal property of the conformal metric $\rho_{\gamma}=\left|\operatorname{grad} u_{\gamma}\right|$ corresponding to a result of Wolontis [17] and Strebel [18] is proved, which connects $\mu_{\gamma}$ with the extremal length of a certain family of curves on $S$. As an application, a characterization of a parabolic boundary component is obtained in terms of conformal metrics. Another characterization of a parabolic boundary component is given by means of the divergence of a modular series $\sum \mu\left(E_{n} ; \gamma_{n-1}, \gamma_{n}\right)$. The sufficient part of this theorem implies the modular criterion of Savage [15]. A theorem shows the equivalence of perimeter in Ahlfors and Beurling's sense and capacity in Sario's sense.

Section 4 deals with the class $M_{\gamma}$ of Riemann surfaces for which all $\gamma$ are parabolic in the case of a finite genus. The conformal mapping properties of $u_{\gamma}$ and $t_{\gamma}$ are discussed, and, for planar Riemann surfaces, the equalities $O_{S B}=M_{\gamma}=O_{S D}[1,14]$ are proved. Finally a theorem is proved which shows the connection between $M_{\gamma}$ and the class of Riemann surfaces for which the continuation is topologically unique, or which do not possess essential continuations.

\section{§2. HaRmonic Functions and Modulus}

2.1. Moduli of a compact subregion. Let $S_{0}$ denote a relatively compact subregion of a Riemann surface $R$. We assume that the boundary 
of $S_{0}$ is a set $\gamma_{0} \cup \alpha_{0}$, where $\gamma_{0}$ is a closed analytic Jordan curve and $\alpha_{0}$ consists of a finite number of closed analytic Jordan curves $\alpha_{01}, \cdots, \alpha_{0 k}$ $(k \geqq 1)$. We assign to each $\alpha_{0 i}(i=1, \cdots, k)$ as positive orientation the positive sense with respect to $S_{0}$ and to $\gamma_{0}$ the sense for which $\gamma_{0}$ and $\alpha_{0}$ are homologous.

If $u$ is a harmonic function on $S_{0}$ then we denote the conjugate period of $u$ around $\alpha_{0 i}$ by $p_{i}(u)$. This is defined by the integral $\int_{\alpha_{0 i}^{\prime}} d \bar{u}$, where $\alpha_{0 i}^{\prime}$ is any closed Jordan curve on $S_{0}$ such that $\alpha_{0 i}$ and $\alpha_{0 i}^{\prime}$ are homologous. If $u$ is harmonic on $S_{0} \cup \alpha_{0 i}$ then clearly $p_{i}(u)=\int_{\alpha_{0 i}} d \bar{u}$. The period vector $\left(p_{1}(u), \cdots, p_{k}(u)\right)$ will be denoted by $p(u)$.

Lemma 1. There is a harmonic function $u_{0}=u\left(z ; S_{0} ; \gamma_{0}, k_{01}\right)$ on $S_{0}$ satisfying the following conditions:

(a) $u_{0}=0$ on $\gamma_{0}$ and $u_{0}=\mu_{0 i}=$ const. on $\alpha_{0 i}(i=1, \cdots, k)$,

(b) $p\left(u_{0}\right)=(1,0, \cdots, 0)$.

(c) $0<u_{0}(z)<\mu_{01}$ on $S_{0}$ and on the boundary curves $\alpha_{02}, \cdots, \alpha_{0 k}$.

Proof. Denote the harmonic measure of $\alpha_{0 i}$ with respect to $S_{0}$ by $\omega_{i}$, and consider the function

$$
u(z)=\sum_{i=1}^{k} \mu_{i} \omega_{i}(z),
$$

where $\mu_{i}$ are arbitrary real numbers. Clearly, this function is harmonic on $\overline{S_{0}}=S_{0} \cup \gamma_{0} \cup \alpha_{0}$. Setting $a_{i j}=p_{i}\left(\omega_{i}\right)$, we obtain

$$
p_{i}(u)=\int_{\alpha_{0 i}} d \bar{u}=\sum_{j=1}^{k} a_{i j} \mu_{j} .
$$

We assert that this linear mapping of the $k$-dimensional cartesian space into itself is one-to-one. In fact, from Green's formula

$$
D(u) \equiv D\left(u ; S_{0}=\sum_{i=1}^{k} \int_{\alpha_{0 i}} u d \bar{u}=\sum_{i=1}^{k} \mu_{i} p_{i}(u),\right.
$$

we see that the condition $p_{i}(u)=0$, for all $i$, implies $D(u)=0$, that is $u \equiv 0$ (since $u=0$ on $\gamma_{0}$ ) and consequently $\mu_{i}=0$, for all $i$, which proves our assertion. Hence we deduce in particular that the above linear mapping is onto, i.e., for any $p$, there is a function $u=\sum \mu_{i} \omega_{i}(z)$ such that $p(u)=p$. Let $u_{0}$ denote the function (1.1) corresponding to $p_{0}=$ $(1,0, \cdots, 0)$. This is clearly the unique bounded harmonic function on $S_{0}$ satisfying (a) and (b).

Now denote the maximum and the minimum of $u_{0}$ on the boundary of $S_{0}$ by $M_{0}$ and $m_{0}$ respectively. From the maximum principle, we have 
$m_{0}<u_{0}(z)<M_{0}$ on $S_{0}$. It follows that $\partial u_{0} / \partial n \leqq 0$ on each boundary curve $\gamma\left(M_{0}\right)$ on which $u_{0}(z)=M_{0}$. Here $\partial / \partial n$ denotes the derivative in the direction of the interior normal. Since $u_{0}$ is not constant and $\partial u_{0} / \partial n$ is continuous, there exists a subarc of $\gamma\left(M_{0}\right)$ on which $\partial u_{0} / \partial n<0$ and therefore

$$
\int_{\gamma(M))} d \bar{u}_{0}=-\int_{\gamma(M U)} \frac{\partial u_{0}}{\partial n}|d z|>0,
$$

where $\gamma\left(M_{0}\right)$ is described in the positive sense with respect to $S_{0}$. This and condition (b) implies that $\gamma\left(M_{0}\right)$ coincides necessarily with $\alpha_{01}$, whence $M_{0}=\mu_{01}$ and this maximum is attained only on $\alpha_{01}$. Similarly, it can be proved that $m_{0}=0$ and that this minimum is attained only on $\gamma_{0}$ This completes the proof of Lemma 1 .

Lemma 2. The function $u_{0}$ gives the minimum of $D(u)$,

$$
\min D(u)=D\left(u_{0}\right) \text {, }
$$

in the class of all harmonic functions $u$ on $S_{0}$ with $u=0$ on $\gamma_{0}$ and $p(u)=$ $(1,0, \cdots, 0)$.

Proof. Clearly, the function $u_{0}$ belongs to the class of admissible functions and, by Green's formula,

$$
D\left(u_{0}\right)=\sum_{i=1}^{k} \mu_{0 i} p_{i}\left(u_{0}\right)=\mu_{01}<\infty .
$$

Let $u$ be any admissible function with $D(u)<\infty$. Setting $u-u_{0}=h$, we have

$$
D(u)=D\left(u_{0}\right)+D(h)+2 D\left(u_{0}, h\right),
$$

where $D\left(u_{0}, h\right)=D\left(u_{0}, h ; S_{0}\right)$ is the mixed Dirichlet integral of $u_{0}$ and $h$ over $S_{0}$. We shall show that $D\left(u_{0}, h\right)=0$. If $u$ is harmonic on $\bar{S}_{0}$ then Green's formula gives immediately

$$
D\left(u_{0}, h\right)=\int_{\alpha_{0}} u_{0} d \bar{h}=\sum_{i=1}^{k} \mu_{0 i} p_{i}(h)=0
$$

since, for all $i, p_{i}(h)=p_{i}(u)-p_{i}\left(u_{0}\right)=0$. If the above assumption is not true, we consider the open set $S_{0}(\varepsilon)=S_{0}-\cup_{i=1}^{k} E_{0 i}(\varepsilon)$, where $\varepsilon$ is a positive number, sufficiently small, and $E_{\mathrm{c} i}(\varepsilon)$ is the set (of points of $S_{0}$ for which) $\mu_{0 i}-\varepsilon \leqq u_{0}(z) \leqq \mu_{0 i}+\varepsilon$. The boundary of $S_{0}(\varepsilon)$ consists only of level lines of $u_{0}$. On the other hand each level line $c(\mu): u_{0}(z)=\mu\left(0<\mu<\mu_{01}\right.$, $\left.\mu \neq \mu_{0 i}, i=1, \cdots, k\right)$ is a dividing cycle on $S_{0}$ (that is, $c(\mu)$ is homologous with a sum of $\alpha_{0 i}$ ) and therefore $\int_{c(\mu)} d \bar{h}=0$. Hence, Green's formula gives again $D\left(u_{0}, h ; S_{0}(\varepsilon)\right)=0$ and, as $\varepsilon \rightarrow 0, D\left(u_{0}, h\right)=0$. We conclude finally that 


$$
D(u)=D\left(u_{0}\right)+D\left(u-u_{0}\right),
$$

which proves our lemma.

The uniqueness of the minimizing function $u_{0}$ is an immediate consequence of (2.2). For, if $D(u)=D\left(u_{0}\right)$, we conclude from (2.2) that $D\left(u-u_{0}\right)=0$, that is $u \equiv u_{0}$, since $u-u_{0}=0$ on $\gamma_{0}$.

The function $u_{0}=u\left(z ; S ; \gamma_{0}, \alpha_{01}\right)$ will be called the extremal function of $S_{0}$ for $\gamma_{0}$ and $\alpha_{01}$. The quantity $\mu_{01}=D\left(u_{0}\right)$ will be called the modulus of $S_{0}$ for $\gamma_{0}$ and $\alpha_{01}$ and denoted generally by $\mu\left(S_{0} ; \gamma_{0}, \alpha_{01}\right)$.

2.2. Modulus of a boundary component. Let us consider a boundary component $\gamma$ of an open Riemann surface $R$, and let $S$ be a given neighborhood of $\gamma$. Let $\gamma_{0}$ be the relative boundary of $S$ (see 1.1). An exhaustion of $S$ is a sequence $\left\{S_{n}\right\}(n=1,2, \cdots)$ of subregions of $R$ such that: (1) $S_{n}$ is a relatively compact subregion of $R$ and the relative boundary of $S_{n}$ is a set $\gamma_{0} \cup \alpha_{n}$, where $\gamma_{0} \cap \alpha_{n}=0$ and $\alpha_{n}$ consists of a finite number of closed analytic Jordan curves $\alpha_{n i}$, (2) $S_{n} \subset S_{n+1}$, $\bigcup_{n+1}^{\infty} S_{n}=S$, and (4) each connected component of $S-S_{n}$ is non-compact and its relative boundary consists of a single $\alpha_{n i}$. We assign to each $\alpha_{n i}$ as positive orientation the positive sense with respect to $S_{n}$ and to $\gamma_{0}$ the sense for which $\gamma_{0}$ and $\alpha_{n}$ are homologous.

Let $\gamma_{n}$ be the curve $\alpha_{n i}$ which separates $\gamma$ from $\gamma_{0}$, and let $\{n\}_{\gamma}$ be the class of all harmonic functions $u$ on $S$ with $u=0$ on $\gamma_{0}$ and

$$
\int_{\gamma_{n}} d \bar{u}=1 \text { and } \int_{a_{n i} \neq \gamma_{n}} \mathrm{~d} \bar{u}=0
$$

for all $n$. It is easy to see, using Green's formula, that conditions (2.3) are independent of the particular exhaustion which is used.

THEOREM 2. In $\{u\}_{\gamma}$ there exists a function $u_{\gamma}$ with the property

$$
\min D(u ; S)=D\left(u_{\gamma} ; S\right) .
$$

Moreover, for any $u$,

$$
D(u ; S)=D\left(u_{\gamma} ; S\right)+D\left(u-u_{\gamma} ; S\right) .
$$

Proof. Denote by $u_{n}$ the extremal function of $S_{n}$ for $\gamma_{0}$ and $\gamma_{n}$, and put $\mu_{n}=D\left(\mu_{n} ; S_{n}\right)=$ value of $u_{n}$ on $\gamma_{n} ; \mu_{n}$ is the modulus of $S_{n}$ for $\gamma_{0}$ and $\gamma_{n}$.

Since the restriction of $u_{n+1}$ to $S_{n}$ satisfies the condition of Lemma 2 (where $S_{0}$ is replaced by $S_{n}$ and $\alpha_{01}$ by $\gamma_{n}$ ), we have

$$
\mu_{n}=D\left(u_{n} ; S_{n}\right) \leqq D\left(u_{n+1} ; S_{n}\right) \leqq D\left(u_{n+1} ; S_{n+1}\right)=\mu_{n+1} .
$$

Similarly, we see that $\mu_{n} \leqq \mu_{\gamma}$, where $\mu_{\gamma}$ is the greatest lower bound of 
$D(u ; S)$ for $u$ in $\{u\}_{\gamma}$. Thus, $\lim _{n \rightarrow \infty} \mu_{n}$ exists and we have

$$
\lim _{n \rightarrow \infty} \mu_{n} \leqq \mu_{\gamma} .
$$

For a fixed $N$, let $s$ be the bounded harmonic function on $S_{N}$ with $s=0$ on $\gamma_{0}$ and $s=d$ on $\alpha_{N}$, where $d$ is a constant value determined by $\int_{\alpha_{N}} d \bar{s}=1$. From Green's formula $\left.\int_{\alpha_{N}} u_{n} d \bar{s}-s d \bar{u}_{n}\right)=0$ and the boundary behavior of $u_{n}$ and $s$, we obtain

$$
\int_{\alpha_{N}} u_{n} d \bar{s}=d
$$

for all $n \geqq N$, whence $\min _{\alpha_{N}} u_{n} \leqq d$. It follows from Harnack's principle that the sequence $\left\{u_{n}\right\}$ is compact. A subsequence, say again $\left\{u_{n}\right\}$, converges, uniformly on each $S_{N}$, to a function $u$. Obviously this function belongs to $\{u\}_{\gamma}$, so that

$$
\mu_{\gamma} \leqq D\left(u_{\gamma} ; S\right)
$$

On the other hand, the lower semicontinuity of the Dirichlet integral gives

$$
D\left(u_{\gamma} ; S\right) \leqq \lim D\left(u_{n} ; S_{n}\right)=\lim \mu_{n} .
$$

From the three preceding inequalites we conclude that

$$
D\left(u_{\gamma} ; S\right)=\lim \mu_{n}=\mu_{\gamma},
$$

which proves the first assertion of Theorem 2 .

Let us now prove equality (2.4), for any $u$ in $\{u\}_{\gamma}$. This is evident if $D(u ; S)=\infty$. Suppose $D(u ; S)<\infty$, and put $u-u_{\gamma}=h$. For any real number $\varepsilon, u_{\gamma}+\varepsilon h \in\{u\}_{\gamma}$, and therefore

$$
D\left(u_{\gamma}+\varepsilon h\right)=D\left(u_{\gamma}\right)+2 \varepsilon D\left(u_{\gamma}, h\right)+\varepsilon^{2} D(h) \geqq D\left(u_{\gamma}\right) .
$$

Since $D\left(u_{\gamma}+\varepsilon h\right)<\infty$, this is possible only if $D\left(u_{\gamma}, h\right)=0$, so that, as $\varepsilon=1$, we obtain (2.4).

As in Lemma 2, the uniqueness of the minimizing function $u_{\gamma}$ in the case $\mu_{1}<\infty$ is an immediate consequence of (2.4).

The function $u_{\gamma}$ will be called the extremal function of $S$ for $\gamma_{0}$ and $\gamma$ and denoted generally by $u\left(z ; S ; \gamma_{0}, \gamma\right)$. The conformal invariant $\mu_{1}=$ $D\left(u_{\gamma}, S\right)$ will be called the modulus of $S$ for $\gamma_{0}$ and $\gamma$ or, simply, for fixed $S$, the modulus of $\gamma$. It will be denoted generally by $\mu\left(S ; \gamma_{0}, \gamma\right)$.

2.3. Parabolic boundary components. Let $\gamma$ be a boundary component of an open Riemann surface $R$. Consider any two neighborhoods $S$ and $S^{\prime}$ of $\gamma$, and denote by $\gamma_{0}$ and $\gamma_{0}^{\prime}$ the relative boundaries of $S$ and 
$S^{\prime}$ respectively. Set $u\left(z ; S ; \gamma_{0}, \gamma\right)=u_{\gamma}, u\left(z ; S^{\prime} ; \gamma_{0}^{\prime}, \gamma\right)=u_{\gamma}^{\prime}, \mu\left(S ; \gamma_{0}, \gamma\right)=$ $\mu_{\gamma}, \mu\left(S^{\prime} ; \gamma_{0}^{\prime}, \gamma\right)=\mu^{\prime}{ }_{\gamma}$.

Lemma 3. The moduli $\mu_{\gamma}$ and $\mu_{\gamma}^{\prime}$ are simultaneously finite or infinite.

Proof. Suppose first $S \subset S^{\prime}$, and let $\left\{S_{n}^{\prime}\right\}$ be an exhaustion of $S^{\prime}$. The regions $S_{n}=S \cap S_{n}^{\prime}$ give, for $n$ sufficiently large, an exhaustion of $S$. Set $u\left(z ; \gamma_{0}, \gamma_{n}\right)=u_{n}, u\left(z ; S_{n}^{\prime} ; \gamma_{0}^{\prime}, \gamma_{n}\right)=u_{n}^{\prime}, \mu\left(S_{n} ; \gamma_{0}, \gamma_{n}\right)=\mu_{n}, \mu\left({S^{\prime}}_{n} ; \gamma_{0}^{\prime}, \gamma_{n}\right)=$ $\mu_{n}^{\prime}$.

From Green's formula

$$
\int_{\alpha_{n} \mathrm{U} \gamma_{0}^{-1}}\left(u_{n}^{\prime} d \bar{u}_{n}-u_{n} d \bar{u}_{n}^{\prime}\right)=0
$$

it follows

$$
\mu_{n}^{\prime}-\mu_{n}=\int_{\gamma_{0}} u_{n}^{\prime} d \bar{u}_{n}
$$

Hence, as $n \rightarrow \infty$, we obtain

$$
\mu_{\gamma}^{\prime}-\mu_{\gamma}=\int_{\gamma_{0}} u_{\gamma}^{\prime} d \bar{u}_{\gamma} .
$$

This proves our lemma in the particular case $S \subset S^{\prime}$.

Let us now consider the general case, and construct a third neighborhood $S^{\prime \prime}$ of $\gamma$ such that $S^{\prime \prime} \subset S \cap S^{\prime}$. Let $\gamma^{\prime \prime}{ }_{0}$ denote the relative

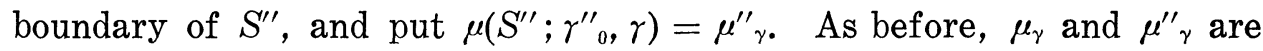
simultaneously finite or infinite. The same is valid for $\mu_{\gamma}^{\prime}$ and $\mu^{\prime \prime}{ }_{\gamma}$ and consequently for $\mu_{\gamma}$ and $\mu_{\gamma}^{\prime}$, which completes the proof of Lemma 3 .

A boundary component $\gamma$ of $R$ is called parabolic if $\mu_{\gamma}=\infty$ and hyperbolic if $\mu_{\gamma}<\infty$. From Lemma 3, this condition is independent of the neighborhood $S$ which is used, i.e. the parabolicity of a $\gamma$ is a $\gamma$ property of $R$. The class of all Riemann surfaces for which all boundary components are parabolic will be denoted by $M_{\gamma}$. The property $R \in M_{\gamma}$ (or $R \notin M_{\gamma}$ ) is a boundary property of $R$.

Consider now the capacity function $t_{\gamma}$ of $R$ for $\gamma$ with respect to a fixed parametric disc $|z| \leqq 1$. Let $\lambda$ denote a positive number which is sufficiently small such that the level line $c(\lambda): t_{\gamma}(z)=\log \lambda$ is a closed Jordan curve and the set $t_{\gamma}(z) \leqq \log \lambda$ is compact. The set $S(\lambda): t_{\gamma}(z)>\log \lambda$ is then a neighborhood of $\gamma$. Put $u(z ; S(\lambda) ; c(\lambda), \gamma)=u_{\gamma, \lambda}, \mu(S(\lambda) ; c(\lambda), \gamma)=$ $\mu_{\gamma, \lambda}$.

LEMMA 4. If $\lambda$ satisfies the above conditions, then

$$
t_{\gamma}(z)-\log \lambda=2 \pi u_{\gamma, \lambda}(z),
$$


and

$$
k_{\gamma}-\log \lambda=2 \pi \mu_{\gamma, \lambda}
$$

Proof. Consider an exhaustion $\left\{R_{n}\right\}$ of $R$ as in 2.1. The regions $S_{n}(\lambda)=R_{n} \wedge S(\lambda)$ give, for $n$ sufficiently large, an exhaustion of $S(\lambda)$. Set $u\left(z ; S_{n}(\lambda) ; c(\lambda), \gamma_{n}\right)=u_{n, \lambda}, \mu\left(S_{n}(\lambda) ; c(\lambda), \gamma_{n}\right)=\mu_{n, \lambda}, t,-2 \pi u_{\gamma, \lambda}=h, t_{n}-2 \pi u_{n \pi}=$ $h_{n}$, where $t_{n}$ is the function on $R_{n}$ defined in 1.2. From Green's formula, we have

$$
D\left(h_{n} ; S_{n}(\lambda)\right)=\int_{\beta n} h_{n} d \bar{h}_{n}-\int_{c(\lambda)} h_{n} d \bar{h}=-\int_{c(\lambda)} h_{n} d \bar{h}_{n}
$$

since $h_{n}=$ const. on $\beta_{n i}$ and $\int_{\beta_{n i}} d \bar{h}_{n}=0$, for all $\beta_{n i}$. Hence, by the lower semicontinuity of the Dirichlet integral,

$$
D(h ; S(\lambda)) \leqq-\int_{c(\lambda)} h d \bar{h}=0
$$

since $h=$ const. $=\log \lambda$ on $c(\lambda)$ and $\int_{c(\lambda)} d \bar{h}=0$. We conclude that $h \equiv$ $\log \lambda$, which proves (2.5).

Now apply Green's formula on $S_{n}(\lambda)$ to $u_{n, \lambda}$ and $t_{n}$. We obtain

$$
k_{n}-2 \pi \mu_{n, \lambda}=\int_{c(\lambda)} t_{n} d \bar{u}_{n, \lambda}
$$

whence, as $n \rightarrow \infty$,

$$
k_{\gamma}-2 \pi \mu_{\gamma, \lambda}=\int_{c(\lambda)} t_{\gamma} d \bar{u}_{\gamma, \lambda}=\log \lambda
$$

which completes the proof of Lemma 4 .

THEOREM 3. A boundary component $\gamma$ of $R$ is parabolic if and only if it has a vanishing capasity.

Proof. This is evident from Lemmas 3 and 4.

Corollary. $\quad M_{\gamma}=C_{n}$.

\section{§3 Modulus and Conformal Metrics}

3.1. Definitions. Consider a non-negative function $\rho(z)$ which is defined on each parametric disc $K_{z}:|z| \leqq 1$ of a subregion $S$ of $R$ and satisfies

$$
\rho(z)=\rho\left(z^{\prime}\right)\left|\begin{array}{c}
d z^{\prime} \\
d z
\end{array}\right|
$$


at corresponding points $z, z^{\prime}$ of any two overlapping $K_{z}$ and $K_{z^{\prime}}$. We say that $\rho$ is a conformal metric on $S$. We define the $\rho$-length of any cycle $c$ (finite set of closed Jordan curves) on $S$ by the lower Darboux integral (see [4])

$$
l(\rho ; c)=\int_{c} \rho(z)|d z|
$$

A conformal metric $\rho$ is said to be measurable on $S$ if its restriction to any parametric disc is measurable in Lebesgue's sense. If $\rho$ is a measurable conformal metric on $S$, we define the $\rho$-area of $S$ by the Lebesgue integral

$$
\mathrm{A}(\rho ; S)=\int_{S} \rho^{2}(z) d \sigma_{z},
$$

where $\sigma_{z}$ is the Lebesgue measure on $K_{z}$. A measurable conformal metric $\rho$ defined on $S$ is said to be $A$-bounded on $S$ if $A(\rho ; S)<\infty$.

3.2. Extremal conformal metrics. Consider first the relatively compact subregion $S_{0}$ of 2.1. We prove the following

LEMMA 5. The conformal metric $\rho_{0}=\left|\operatorname{grad} u_{0}\right|$ gives the minimum of $A\left(\rho ; S_{0}\right)$,

$$
\min A\left(\rho ; S_{0}\right)=A\left(\rho_{0} ; S_{0}\right),
$$

in the class of all conformal metrics satisfying $l(\rho ; c) \geqq 1$, for all dividing cycles $c$ on $S_{0}$ which separate $\alpha_{01}$ from $\gamma_{0}$.

Moreover, for any admissible $\rho$,

$$
A\left(\rho ; S_{0}\right) \geqq A\left(\rho_{0} ; S_{0}\right)+A\left(\rho-\rho_{0} ; S_{0}\right) .
$$

Proof. Clearly the conformal metric $\rho_{0}$ satisfies the condition of the lemma, and $A\left(\rho_{0} ; S_{0}\right)=D\left(u_{0} ; S_{0}\right)=\mu_{01}<\infty$. Let $\rho$ be any admissible conformal metric on $S_{0}$ with $A\left(\rho ; S_{0}\right)<\infty$.

We evaluate the integral

$$
\int_{S_{0}} \rho(z) \rho_{0}(z) d \sigma_{z} .
$$

Take $w_{0}=u_{0}+i \bar{u}_{0}$ for the local parameter on $S_{0}$, so that $\rho_{0}\left(w_{0}\right) \equiv 1$. Denote the level line $u_{0}(z)=\mu\left(0 \leqq \mu \leqq \mu_{01}\right.$; see Lemma 1$)$ by $c(\mu)$. From Fubini's theorem,

$$
\int_{s_{0}} \rho(z) \rho_{0}(z) d \sigma_{z}=\int_{0}^{\mu_{01}} d \mu \int_{c(\mu)} \rho\left(w_{0}\right) d \bar{u}_{0} .
$$


Here the integral $\int_{c(\mu)} \rho\left(w_{0}\right) d \vec{u}_{0}$ exists almost everywhere, for $\mu$ on the closed interval $\left[0, \mu_{01}\right]$. But $c(\mu)$ is, for any $\mu \neq \mu_{0 i}$, a dividing cycle on $S_{0}$ which separates $\alpha_{01}$ from $\gamma_{0}$ and therefore, almost everywhere,

$$
\int_{c(\mu)} \rho\left(w_{0}\right) d \bar{u}_{0}=\int_{c(\mu)} \rho(z)|d z| \geqq \int_{c(\mu)} \rho(z)|d z| \geqq 1
$$

From the two preceding relations it follows that

$$
\int_{S_{0}} \rho(z) \rho_{0}(z) d \sigma_{z} \geqq \mu_{01}
$$

Now put $\rho=\rho_{0}+\left(\rho-\rho_{0}\right)$ in $A\left(\rho ; S_{0}\right)$; we obtain

$$
A\left(\rho ; S_{0}\right)=\mu_{01}+A\left(\rho-\rho_{0} ; S_{0}\right)+2 \int_{S_{0}} \rho \rho_{0} d \sigma-2 \mu_{01}
$$

and, from the preceding inequality, we conclude finally that

$$
A\left(\rho ; S_{0}\right) \geqq \mu_{01}+A\left(\rho-\rho_{0} ; S_{0}\right),
$$

which proves our lemma.

Clearly the admissible conformal metric which minimizes $A\left(\rho ; S_{0}\right)$ is unique. For, if $A\left(\rho ; S_{0}\right)=A\left(\rho_{0} ; S_{0}\right)=\mu_{01}<\infty$, we deduce from (3.2) that $A\left(\rho-\rho_{0} ; S_{0}\right)=0$, i.e. $\rho=\rho_{0}$ almost everywhere on $S_{0}$.

Now let $\gamma$ be a boundary of $R$, and let $S$ be a given neighborhood of $\gamma$. Let $\{\rho\}_{\gamma}$ denote that class of all measurable conformal metrics defined on $S$ which satisfy the condition

$$
l(\rho ; c) \geqq 1,
$$

for all dividing cycles $c$ which separate $\gamma$ from $\gamma_{v}$. If $u \in\{u\}_{\gamma}$, then obviously $|\operatorname{grad} u| \in\{\rho\}_{\gamma}$. This is valid, in particular, for the conformal metric $\rho_{\gamma}=\left|\operatorname{grad} u_{\gamma}\right|$. The $\rho_{\gamma}$-area of $S$ is $A\left(\rho_{\gamma} ; S\right)=D\left(u_{\gamma} ; S\right)=\mu_{\gamma}$.

THEOREM 4. In $\left\{\rho_{\gamma}\right.$ the conformal metric $\rho_{\gamma}=\left|\operatorname{grad} u_{\gamma}\right|$ gives the minimum of $A(\rho ; S)$ :

$$
\min A(\rho ; S)=A\left(\rho_{\gamma} ; S\right)
$$

Moreover, for any $\rho$,

$$
A(\rho ; S) \geqq A\left(\rho_{\gamma} ; S\right)+A\left(\rho-\rho_{\gamma} ; S\right) .
$$

Proof. If $A(\rho ; S)=\infty$, (3.5) is evident. Assume now that there exists in $\{\rho\}_{\gamma}$ a conformal metric $\rho$ which is $A$-bounded.

Set $\left|\operatorname{grad} u_{n}\right|=\rho_{n}$ (see 2.2). Since $A(\rho ; S) \geqq A\left(\rho ; S_{n}\right)$, we conclude from Lemma 5 that 


$$
A(\rho ; S) \geqq \mu_{n}+A\left(\rho-\rho_{n} ; S_{n}\right)
$$

As $n \rightarrow \infty$, Fatou's Lemma gives immediately

$$
A(\rho ; S) \geqq \mu_{\gamma}+\liminf A\left(\rho-\rho_{n} ; S_{n}\right) \geqq \mu_{\gamma}+A\left(\rho-\rho_{\gamma} ; S\right),
$$

which proves (3.5) and the theorem.

As in Lemma 5, the uniqueness of the minimizing conformal metric $\rho_{\gamma}$ in the case $\mu_{\gamma}<\infty$ is an immediate consequence of (3.5).

By Theorem 4, the quantity $\lambda_{\gamma}=\mu_{\gamma}{ }^{-1}$ is equal to the extremal length of the family of all dividing cycles $c$ on $S$ separating $\gamma$ from $\gamma_{0}$ ([1], [5]).

3.3. Parabolic boundary components. We return to the condition $\mu_{\gamma}=\infty$ studied in 2.2.

THEOREM 5. A boundary component $\gamma$ of $R$ is parabolic if and only if, for any neighborhood $S$ of $r$ and for any A-bounded conformal metric $\rho$ on $S$, there exists $a$ dividing cycle separating $\gamma$ from $\gamma_{0}$ with an arbitrarily small $\rho$-length.

Proof. If $\mu_{\gamma}<\infty$, the conformal metric $\rho_{\gamma}$ is $A$-bounded and satisfies $l(\rho ; c) \geqq 1$, for all dividing cycles separating $\gamma$ from $\gamma_{0}$. Conversely, if there is an $A$-bounded conformal metric $\rho$ on $S$ satisfying $l(\rho ; c) \geqq \varepsilon>0$, for all dividing cycles $c$ separating $\gamma$ from $\gamma_{0}$, the conformal metric $\rho^{*}=(1 / \varepsilon) \rho$ is $A$-bounded and belongs to $\{\rho\}_{\gamma}$. Therefore, by Theorem 4 , $\mu_{\gamma}<\infty$.

Theorem 6. Suppose $R$ is imbedded in a larger Riemann surface $R^{*}$. If a boundary component $\gamma$ of $R$ or a part of $\gamma$ realized on $R^{*}$ contains a continuum $\gamma^{*}$, then $r$ is hyperbolic.

Proof. Let $K^{*}:\left|z^{*}\right| \leqq 1$ denote a parametric disc on $R^{*}$ for which $K^{*} \cap \gamma^{*}$ contains a continuum, say again $\gamma^{*}$. Since $\gamma^{*}$ is a boundary continuum of $R$, there exists a disc $\bar{R}_{0} \subset K^{*} \cap R$. In $K^{*}$ let $Q=a b a^{\prime} b^{\prime}$ be a rectangle such that its side $a$ is completely interior to $R_{0}$ and its opposite sides $b, b^{\prime}$ have common points with $r^{*}$.

Set $R-\bar{R}_{0}=S$. We define a conformal metric $\rho_{0}$ on $S$ by setting $\rho_{0}\left(z^{*}\right)=1$ on $Q \cap S$ and $\rho_{0}=0$ otherwise. Clearly $\rho_{0}$ is $A$-bounded and satisfies $l\left(\rho_{0} ; c\right) \geqq l_{0}>0$, where $l_{0}$ is the length of $a$ in $K^{*}$ and $c$ is any dividing cycle separating $\gamma$ from $\gamma_{0}$. Hence, by Theorem $5, \gamma$ is not parabolic.

Let $S$ be a given neighborhood of a boundary component $\gamma$ of $R$, and let $\left\{S_{n}\right\}$ be an exhaustion of $S$ as in 2.2. Let $E_{n}$ denote the connected component of $S_{n}-S_{n-1}$ whose boundary includes $\gamma_{n-1}$ and $\gamma_{n}$. We assert that 


$$
\mu\left(S ; \gamma_{0}, \gamma\right) \geqq \sum_{n=1}^{\infty} \mu\left(E_{n} ; \gamma_{n-1}, \gamma_{n}\right)
$$

In fact, since the restriction of $\rho_{\gamma}$ to $E_{n}$ is admissible in Lemma 5 (where $S_{0}$ is replaced by $E_{n}, \gamma_{0}$ and $\alpha_{01}$ by $\gamma_{n-1}$ and $\gamma_{n}$ respectively), we conclude that $A\left(\rho_{\gamma} ; E_{n}\right) \geqq \mu\left(E_{n} ; \gamma_{n-1}, \gamma_{n}\right)$. Therefore, $\mu\left(S ; \gamma_{0}, \gamma\right) \geqq \sum_{n=1}^{\infty} A\left(\rho_{\gamma} ; E_{n}\right) \geqq$ $\sum_{n=1}^{\infty} \mu\left(E_{n} ; \gamma_{n-1}, \gamma_{n}\right)$, which proves (3.6).

Similarly, it may be proved that

$$
\mu\left(S ; \gamma_{0}, \gamma\right) \geqq \mu\left(E_{1} ; \gamma_{0}, \gamma_{1}\right)+\mu\left(S_{1}^{*} ; \gamma_{1}, \gamma\right),
$$

where $S^{*}{ }_{1}$ is the connected component of $S-\bar{S}_{1}$ whose relative boundary is $\gamma_{1}$.

THEOREM 7. A boundary component $\gamma$ of $R$ is parabolic if and only if there exists an exhaustion of $S$ for which

$$
\sum_{n=1}^{\infty} \mu\left(E_{n} ; \gamma_{n-1}, \gamma_{n}\right)=\infty
$$

Proof. By (3.6), the condition (3.8) is sufficient for the parabolicity of $\gamma$.

Conversely, assume that $\gamma$ is parabolic, and let $\left\{S_{n}\right\}$ be a given exhaustion of $S$. Since

$$
\lim _{n \rightarrow \infty} \mu\left(S_{n} ; \gamma_{0}, \gamma_{n}\right)=\mu\left(S ; \gamma_{0}, \gamma\right)=\infty,
$$

we can choose $n_{1} \geqq 1$ such that $\mu\left(S_{n} ; \gamma_{0}, \gamma_{n_{1}}\right) \geqq 1$. Let $S^{*}{ }_{n_{1}}$ denote the connected component of $S-\bar{S}_{n_{1}}$ whose relative boundary is $\gamma_{n_{1}}$. $S^{*}{ }_{n_{1}}$ is a neighborhood of $\gamma$. Since $\gamma$ is parabolic, we have

$$
\lim _{n \rightarrow \infty} \mu\left(S_{n_{1}, n}^{*} ; \gamma_{n_{1}}, \gamma_{n}\right)=\mu\left(S_{n_{1}}^{*} ; \gamma_{n_{1}}, \gamma\right)=\infty,
$$

where $S_{n_{1}, n}^{*}=S_{n_{1}}^{*} \cap S_{n}$. Therefore, we can choose $n_{2}>n_{1}$ such that $\mu\left(S^{*}{ }_{n_{1}, n_{2}} ; \gamma_{n_{1}}, \gamma_{n_{2}}\right) \geqq 1$. Continuing this procedure, we obtain an exhaustion $\left\{S_{n_{k}}\right\}(k=1,2, \cdots)$ of $S$, which satisfies condition (3.8). Thus Theorem 7 is established.

3.4. Perimeter and capacity. Let $|z| \leqq r_{0}$ be a fixed parametric $\operatorname{disc}$ on $R$, and let $S(r)$ denote the complement of the disc $|z| \leqq r \quad(0<r \leqq$ $r_{0}$ ) with respect to $R$. Set $\mu(S(r) ;|z|=r, \gamma)=\mu_{\gamma, r}$. By (3.7), for $r^{\prime}<r$,

$$
\mu_{\gamma, r^{\prime}} \leqq \frac{1}{2 \pi} \log \frac{r}{r^{\prime}}+\mu_{\gamma, r}
$$


or

$$
-2 \pi \mu_{\gamma, r^{\prime}}-\log r^{\prime} \leqq-2 \pi \mu_{\gamma, r}-\log r
$$

Therefore,

$$
\pi_{\gamma}=\lim _{r \rightarrow 0} \frac{1}{r} e^{-2 \pi \mu_{\gamma, r}}
$$

exists. According to Ahlfors and Beurling [1], we call $\pi_{\gamma}$ perimeter of $\gamma$ with respect to the fixed parametric dics $|z| \leqq r_{0}$. Let $z^{\prime}=\lambda(z)=$ $a z+\cdots, a \neq 0$, be a new local parameter in the neighborhood of the point $P_{0} \in R$ corresponding to $z=0$, and let $\pi^{\prime}{ }_{\gamma}$ denote the perimeter of $\gamma$ with respect to the parametric disc $\left|z^{\prime}\right| \leqq r_{0}^{\prime}$. Set $|z|=r$ and $\left|z^{\prime}\right|=r^{\prime}$. For corresponding $r$ and $r^{\prime}$ by $z^{\prime}=\lambda(z)$, we have

$$
|a| r\left(1-\varepsilon_{r}\right) \leqq r^{\prime} \leqq|a| r\left(1+\varepsilon_{r}\right),
$$

where $\varepsilon_{r}$ is a positive function of $r$ and $\varepsilon_{r} \rightarrow 0$, as $r \rightarrow 0$. It follows, from the conformal invariance and the monotony of modulus, that

$$
\pi_{\gamma}=|a| \pi_{\gamma}^{\prime} .
$$

We now prove the following.

THEOREM 8. If the perimeter $\pi_{\gamma}$ and the capacity $c_{r}$ are defined with respect to the same parametric disc $|z| \leqq r_{0}$, then $\pi_{\gamma}=c_{\gamma}$.

Proof. From (1.6) and (3.9), it is sufficient to prove the required equality for a particular parametric disc of the point $P_{0}$. We choose this parametric disc, say again $|z| \geqq r_{0}$, such that $t_{\gamma} \equiv \log |z|$ on $|z| \leqq r_{0}$. Then, by (2.6), we conclude immediately that

$$
\pi_{\gamma}=\lim _{\lambda \rightarrow 0} \frac{1}{\lambda} e^{-2 \pi \mu_{\gamma, \lambda}}=e^{-k_{\gamma}}=c_{\gamma},
$$

which proves our theorem.

CoRollary. If $P_{\gamma}$ denote the class of Riemann surfaces defined by $\pi_{\gamma}=0$, for all $\gamma$, then $P_{\gamma}=c_{\gamma}=M_{\gamma}$.

\section{$\S 4$. Riemann Surfaces of Finite Genus}

4.1. Planar subregions. Let $\gamma$ be a boundary component of an open Riemann surface $R$, and suppose that $\gamma$ is hyperbolic and possesses a neighborhood $S$ which is planar.

Set, as usually, $u\left(z ; S ; \gamma_{0}, \gamma\right)=u_{\gamma}, \mu\left(S ; \gamma_{0}, \gamma\right)=\mu_{\gamma}$, and consider the function $w=F_{\gamma}(z)$ defined by 


$$
F_{\gamma}(z)=\exp 2 \pi\left(u_{\gamma}(z)+i \bar{u}_{\gamma}(z)\right)
$$

Consider an exhaustion $\left\{S_{n}\right\}$ of $S$ as in 2.2. Since $S$ is planar, the homology group $H^{1}(S)$ is generated from the boundary curves $\alpha_{n i}$ of $S_{n}(n=1,2, \cdots)$, and we conclude by (2.3) that $F_{\gamma}$ is single-valued. We now prove the following [7]:

THEOREM 9. The function $w=F_{\gamma}(z)$ maps the region $S$ univalently onto the annulus

$$
A_{0, \mu_{\gamma}}: 1<|w|<e^{2 \pi \mu_{\gamma}}
$$

slit along a set of circular arcs around the origin. Here the boundary circumferences $|w|=1$ and $|w| e^{2 \pi \mu \gamma}$ correspond to $\gamma_{0}$ and $\gamma$ respectively. The total area of the slits vanishes.

Proof. We define the function $w=F_{n}(z)$ on $S_{n}$ by

$$
F_{n}(z)=\exp 2 \pi\left(u_{n}(z)+i \vec{u}(z)\right),
$$

where $u_{n}=u\left(z ; S_{n} ; \gamma_{0}, \gamma_{n}\right)$. As before, we see that $F_{n}$ is single-valued, for all $n$.

The function $w=F_{n}(z)$ gives a one-to-one conformal mapping of $S_{n}$ onto the covering surface $S_{n, w}=\left(S_{n}, w=F_{n}(z)\right)$. By the definition of $u_{n},\left|F_{n}(z)\right|$ assumes constant values on the boundary curves of $S_{n}$ and satisfies on $S_{n}$ :

$$
1<\left|F_{n}(z)\right|<e^{2 \pi \mu_{n}} .
$$

It follows that $S_{n, w}$ is an unlimited covering surface of the annulus $A_{0, \mu_{n}}$ slit along a finite number of circular arcs. On the other hand, evaluate the $\rho_{0}$-area of $S_{n, w}$, where

$$
\rho_{0}(w)=\frac{1}{2 \pi|w|}=\frac{1}{2 \pi}\left|\frac{d}{d w} \log w\right| .
$$

Since, for $w=F_{n}(z)$,

$$
\rho_{n}(z)=\left|\operatorname{grad} u_{n}(z)\right|=\frac{1}{2 \pi}\left|\frac{d}{d z} \log w\right|=\rho_{0}(w)\left|\frac{d w}{d z}\right|,
$$

we obtain

$$
A\left(\rho_{0} ; S_{n, w}\right)=A\left(\rho_{n} ; S_{n}\right)=\mu_{n} .
$$

This is equal to the $\rho_{0}$ - area of the annulus $A_{0, \mu_{n}}$. It follows that the covering surface $S_{n, w}$ consists necessarily of a single sheet, that is the function $F_{n}$ is univalent. Since $F_{n} \rightarrow F_{\gamma}$ uniformly on each $S_{N}, F_{\gamma}$ is also univalent. 
Let us now consider the image $S_{w}=F_{\gamma}(z)$. Denote the connected components of the boundary of $S_{w}$ which correspond to $\gamma_{0}$ and $\gamma$ by $\gamma_{w}^{0}$ and $\gamma_{w}$ respectively. Clearly $\gamma_{w}^{0}$ is the circumference $|w|=1$. Further, since $\mu_{n} \leqq \mu_{\gamma}$, for all $n, S_{w}$ is included in the annulus $A_{0, \mu_{\gamma} \cdot}$ As before, the $\rho_{0}$-area of $S_{w}$ is

$$
A\left(\rho_{0} ; S_{w}\right)=A\left(\rho_{\gamma} ; S\right)=\mu_{\gamma},
$$

since

$$
\rho_{\gamma}(z)=\rho_{0}(w)\left|\frac{d w}{d z}\right| \quad\left(w=F_{\gamma}(z)\right)
$$

This is equal to the $\rho_{0}$-area of the annulus $A_{0, \mu_{\gamma^{*}}}$ Accordingly, the complements of $S_{w}$ with respect to $A_{0, \mu_{\gamma}}$ has a (logarithmic and Euclidian) vanishing area.

Assume finally that the set $A_{0, \mu_{\gamma}}-S_{w}$ possesses a connected component $\gamma^{*}{ }_{w}$ which is not a point or a circular arc around the origin. Construct two circumferences $|w|=r_{i}\left(i=1,2 ; r<r_{1}<r_{2}<e^{2 \pi \mu_{\gamma}}\right)$ having common points with $\gamma_{w}^{*}$, and consider a point $w_{0}$ in the annulus $r_{1}<|w|<r_{2}$. Let $K_{\varepsilon}$ be the disc $\left|w-w_{0}\right| \leqq \varepsilon$. Obviously, for $\varepsilon$ sufficiently small, the conformal metric $\rho_{\varepsilon}$, defined by $\rho_{\varepsilon}=0$ on $K_{\varepsilon}$ and $\rho_{\varepsilon}(w)=\rho_{0}(w)$ on $S_{w}-K_{\varepsilon}$, satisfies the condition (3.3), for all dividing cycles $c$ on $S_{w}$ separating $\gamma_{w}$ from $\gamma_{w}^{0}$. This contradicts Theorem 4, since $A\left(\rho_{\varepsilon} ; S_{w}\right)<A\left(\rho_{0} ; S_{w}\right)=\mu$. Therefore, the continuum $\gamma^{*}{ }_{w}$ does not exist. In particular, $\gamma_{w}$ coincides with $|w|=e^{2 \pi \mu} \gamma$. Theorem 9 is completely proved.

4.2. Planar Riemann surfaces. Suppose now that $R$ itself is planar. Let $|z| \leqq 1$ be a fixed parametric disc on $R, \gamma$ a hyperbolic boundary component of $R$, and $c_{\gamma}>0$ the capacity of $\gamma$ with respect to $|z| \leqq 1$. Consider the function $w=T_{\gamma}(z)$ defined by

$$
T_{\gamma}(z)=c_{\gamma} \exp \left(t_{\gamma}(z)+i \bar{t}_{\gamma}(z)\right) .
$$

By Lemma 4 and Theorem 9, we have the following [14]:

THEOREM 10. The function $w=T_{\gamma}(z)$ is univalent and single-valued on $R$ and maps $R$ onto the unit circle slit along a set of circular arcs of vanishing total area. The boundary component $\gamma$ is mapped into the unit circumference.

Let $S B(S D)$ be the class of univalent single-valued analytic functions having a bounded modulus (a finite Dirichlet integral), and let $O_{S B}\left(O_{S D}\right)$ be the class of Riemann surfaces with no functions belonging to $S B(S D)$.

THEOREM 11. [1, 14] For planar Riemann surfaces, 


$$
O_{S B}=M_{\gamma}=O_{S B} .
$$

Proof. Assume first that the planar surface $R$ possesses a hyperbolic boundary component $\gamma$. Then, the function $T_{\gamma}$ of Theorem 10 obviously belongs to the class $S B$ and $S D$.

Conversely, suppose that there exists on $R$ a function $w=T(z)$ which belongs to the class $S B$ or $S D$. In both cases, the image $R_{w}=$ $T(R)$ has a finite Euclidian area. Let $K_{\varepsilon}:\left|w-w_{0}\right| \leqq \varepsilon$ be a disc which is completely included in $R_{w}$. Denote by $r_{w}$ the connected component of the boundary of $R_{w}$ which separates $w=0$ from $w=\infty$ or contains $w=\infty$, The conformal metric $\rho(w)=1 / 2 \pi \varepsilon$ is clearly $A$-boundary on $R_{w}-K_{\varepsilon}$ and satisfies condition (3.3), for all dividing cycles on $R_{w}-K_{\varepsilon}$ which separate $\gamma_{w}$ from $\left|w-w_{0}\right|=\varepsilon$. We conclude that the boundary component $\gamma$ of $R$ which corresponds to $\gamma_{w}$ is hyperbolic.

4.3. Riemann surfaces of finite genus. A continuation of a Riemann surface $R$ is defined by (1) another Riemann surface $R^{\prime}$ and (2) a oneto-one conformal mapping $T: R \rightarrow R^{\prime}, T(R) \subset R^{\prime},[2,4,8,9,11,12]$. If $R^{\prime}$ is a compact Riemann surface, the continuation is called compact. If $R^{\prime}-T(R)$ contains interior points, the continuation is called essential $[9,12]$.

Let $R$ be a Riemann surface of finite genus. We say that the continuation of $R$ is topologically unique if, for any two compact continuations $T_{\nu}: R \rightarrow R_{\nu}^{\prime}(\nu=1,2)$ of $R$, there exists a topological mapping $h^{*}{ }_{12}=R_{1}^{\prime} \rightarrow R_{2}^{\prime}, h_{12}^{*}\left(R_{1}^{\prime}\right)=R_{2}^{\prime}$, with $h_{12}^{*} T_{1}(R)=h_{12}$, where $h_{12}=T_{2} T_{1}^{-1}$. If, in addition, $h^{*}{ }_{12}$ is always a conformal mapping, the continuation of $R$ is said to be conformally unique.

Let $O_{A D}$ denote the class of Riemann surfaces with no non-constant single-valued analytic functions having a finite Dirichlet integral. It is well known that the continuation of a Riemann surface $R$ of finite genus is conformally unique if and only if $R \in O_{A D}[1,8,12]$. We now prove the following

THEOREM 12. For Riemann surfaces of finite genus, the following conditions are equivalent:

(1) $R \in M_{\gamma}$

(2) The continuation of $R$ is topologically unique.

(3) $R$ does not possess an essential continuation.

Proof. (1) $\rightarrow$ (2). If $R \in M_{\gamma}$ and $T_{\nu}: R \rightarrow R^{\prime}{ }_{\nu}(\nu=1,2)$ are compact continuations of $R$, then, by Theorem 6 , the sets $\beta_{\nu}=R_{\nu}^{\prime}-T_{\nu}(R)$ are totally disconnected. Set $T_{2} T_{1}^{-1}=h_{12}$. We define a topological mapping $h^{*}{ }_{12}$ of $R_{1}^{\prime}$ onto $R_{2}^{\prime}$ as follows. First, set $h_{12}^{*}\left(P_{1}\right)=h_{12}\left(P_{1}\right)$, for any $P_{1} \in T_{1}(R)$. Now let $P_{1} \in \beta_{1}$. Since $\beta_{1}$ is totally disconnected, there is 
a fundamental sequence $\left\{U_{n}\right\}$ of neighborhoods of $P_{1}$ such that the open sets $V_{n}=U_{n} \cap T_{1}(R)$ are connected. Set $E\left(P_{1}\right)=\cap_{n} h_{12}\left(V_{n}\right)$. Clearly this is a closed and connected set. On the other hand, $E\left(P_{1}\right) \subset \beta_{2}$ and, since $\beta_{2}$ is totally disconnected $E\left(P_{1}\right)$ contains a single point $P_{2}$. Set $h^{*}{ }_{12}\left(P_{1}\right)=$ $P_{2}$. It is easy to see that $h_{12}^{*}$ is a topological mapping between $R_{1}^{\prime}$ and $R_{2}^{\prime}$.

(2) $\rightarrow(3)$. If $R$ possesses an essential continuation $T_{1}: R \rightarrow R_{1}^{\prime}$, we may construct in an evident manner another compact continuation $T_{2}: R \rightarrow R_{2}^{\prime}$ of $R$ such that $R_{1}^{\prime}$ and $R_{2}^{\prime}$ have different genera.

$(3) \rightarrow(1)$. Assume that $R \notin M$, i.e. $R$ possesses some boundary component $\gamma$ which is hyperbolic. Let $S$ be a neighborhood of $\gamma$. We have $\mu_{\gamma}<\infty$. By Theorem 9, there is a one-to-one conformal mapping of $S$ into the finite annulus $1<|w|<e^{2 \pi \mu_{\gamma}}$. Let $K_{w}$ denote the set $|w|>1$. Clearly the Riemann surface $R^{\prime}=(R-S) \cup K_{w}$ defines an essential continuation of $R$, and therefore $(3) \rightarrow(1)$. Thus, Theorem 12 is established

Corollary 1. For Riemann surfaces of finite genus, we have $O_{A D} \subset M_{\gamma}$.

Note that by a theorem of Ahlfors and Beurling [1] this inclusion is strict.

COROLlary 2. Let $R \in M_{\gamma}-O_{A D}$ and of finite genus. Then there exist two compact continuations $T_{\nu}: R \rightarrow R_{\nu}^{\prime}{ }_{\nu}(\nu=1,2)$ of $R$ such that the corresponding topogical mapping $h_{12}^{*}$ is not a conformal mapping.

In particular, we conclude from Corollary 2 that there exist Pompeiu functions which are univalent (see [3], [10], and [16]).

\section{REFERENCES}

1. L. Ahlfors and A. Beurling, Conformal invariants and function-theoretic null sets, Acta Math. 83 (1950), 101-129

2. S. Bochner, Fortsezung Riemannscher Flächen, Math. Ann., 98 (1928), 406-421.

3. A. Denjoy, Sur les singularités discontinues des fonctions analytiques uniformes, C. R. Acad. Sci. Paris, 149 (1909), 386-388.

4. M. Heins, On the continuation of a Riemann surface, Ann. of Math., 43 (1942), 208-297.

5. J. Hersch, Longueurs extrémales et théorie des fonctions, Comm. Math. Helv., 29 (1954). 301-337.

6. B. Kerékjàrtó, Verlesungen über Topologie, Berlin, 1923.

7. H. Grötzsch, Das Kreisbogenschlitztheorem der konformen Abbildung schlichter Bereiche, Leipziger Ber., 83 (1931), 238-253,

8. A. Mori, A remark on the prolongation of Riemann surfaces of finite genus, J. Math. Soc. Japan, 4 (1952), 27-30.

9. R. Nevanlinna, Uniformisierung, Berlin, 1953.

10. D. Pompeiu, Sur la continuité des fonctions de varlables complexes, Ann. Fac. Sci.

Toulouse, 7 (1905) 265-315. 
11. T. Rado, Über eine nichtfortsetzbare Riemannsche Mannigfaltigkeit, Math. Z., 21 (1924), 1-6.

12. L. Sario, Uber Riemannsche Flächen mit hebbarem Rand, Acad. Sci. Fenn., Ser. AI, 50 (1948).

13. - A linear operator method on arbitrary Riemann surfaces, Trans. Amer. Math. Soc., 72 (1952), 218-295.

14. Capacity of the boundary and of a boundary component, Ann. of Math., 59 (1954), 135-144.

15. N. Savage, Weak boundary components, Duke Math. J., 24 (1957), 79-95.

16. S. Stoilow, Lecons sur les principes topologiques de la théorie des fonctions analytiques, Paris, $2^{\ominus}$ éd. 1956.

18. V. Wolontis, Properties of conformal invariants, Amer. J. Math., 74 (1952), 587606.

19. K. Strebel, Die extremale Distanz zweier Enden einer Riemannschen Fläche, Ann. Acad. Sci. Fenn, Ser. AI, 179 (1955).

Mathematical Institute of the R.P.R. ACademy, Bucare:t 



\section{PACIFIC JOURNAL OF MATHEMATICS}

\section{EDITORS}

\section{David Gilbarg}

Stanford University

Stanford, California

\section{R. A. Beaumont}

University of Washington

Seattle 5, Washington

\author{
A. L. Whiteman
}

University of Southern California Los Angeles 7, California

E. G. Straus

University of California

Los Angeles 24, California

\section{ASSOCIATE EDITORS}

\author{
E. F. BECKENBACH \\ C. E. BURGESS \\ M. HALL \\ E. HEWITT
}
A. HORN
V. GANAPATHY IYER
R. D. JAMES
M. S. KNEBELMAN
L. NACHBIN
I. NIVEN
T. G. OSTROM
H. L. ROYDEN

M. M. SCHIFFER

G. SZEKERES

F. WOLF

K. YOSIDA

\section{SUPPORTING INSTITUTIONS}

\author{
UNIVERSITY OF BRITISH COLUMBIA \\ CALIFORNIA INSTITUTE OF TECHNOLOGY \\ UNIVERSITY OF CALIFORNIA \\ MONTANA STATE UNIVERSITY \\ UNIVERSITY OF NEVADA \\ OREGON STATE COLLEGE \\ UNIVERSITY OF OREGON \\ OSAKA UNIVERSITY \\ UNIVERSITY OF SOUTHERN CALIFORNIA
}

\author{
STANFORD UNIVERSITY \\ UNIVERSITY OF TOKYO \\ UNIVERSITY OF UTAH \\ WASHINGTON STATE COLLEGE \\ UNIVERSITY OF WASHINGTON \\ * * * * \\ AMERICAN MATHEMATICAL SOCIETY \\ CALIFORNIA RESEARCH CORPORATION \\ HUGHES AIRCRAFT COMPANY \\ THE RAMO-WOOLDRIDGE CORPORATION
}

Mathematical papers intended for publication in the Pacific Journal of Mathematics should be typewritten (double spaced), and the author should keep a complete copy. Manuscripts may be sent to any one of the four editors. All other communications to the editors should be addressed to the managing editor, E. G. Straus at the University of California, Los Angeles 24, California.

50 reprints per author of each article are furnished free of charge; additional copies may be obtained at cost in multiples of 50 .

The Pacific Journal of Mathematics is published quarterly, in March, June, September, and December. The price per volume (4 numbers) is $\$ 12.00$; single issues, $\$ 3.50$. Back numbers are available. Special price to individual faculty members of supporting institutions and to individual members of the American Mathematical Society: $\$ 4.00$ per volume; single issues, $\$ 1.25$.

Subscriptions, orders for back numbers, and changes of address should be sent to Pacific Journal of Mathematics, 2120 Oxford Street, Berkeley 4, California.

Printed at Kokusai Bunken Insatsusha (International Academic Printing Co., Ltd.), No. 6, 2-chome, Fujimi-cho, Chiyoda-ku, Tokyo, Japan.

PUBLISHED BY PACIFIC JOURNAL OF MATHEMATICS, A NON-PROFIT CORPORATION

The Supporting Institutions listed above contribute to the cost of publication of this Journal, but they are not owners or publishers and have no responsibility for its content or policies. 


\section{Pacific Journal of Mathematics}

\section{Vol. 8, No. $4 \quad$ June, 1958}

Richard Arens, The maximal ideals of certain functions algebras ........ 641

Glen Earl Baxter, An operator identity ........................... 649

Robert James Blattner, Automorphic group representations ........... 665

Steve Jerome Bryant, Isomorphism order for Abelian groups ............ 679

Charles W. Curtis, Modules whose annihilators are direct summands...... 685

Wilbur Eugene Deskins, On the radical of a group algebra ............ 693

Jacob Feldman, Equivalence and perpendicularity of Gaussian

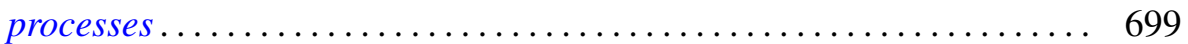

Marion K. Fort, Jr. and G. A. Hedlund, Minimal coverings of pairs by triples....................................... 709

I. S. Gál, On the theory of $(m, n)$-compact topological spaces ......... 721

David Gale and Oliver Gross, A note on polynomial and separable games........................................ 735

Frank Harary, On the number of bi-colored graphs ............... 743

Bruno Harris, Centralizers in Jordan algebras ................... 757

Martin Jurchescu, Modulus of a boundary component ............... 791

Hewitt Kenyon and A. P. Morse, Runs . . . . . . . . . . . . . . . . . . . . . . 811

Burnett C. Meyer and H. D. Sprinkle, Two nonseparable complete metric

spaces defined on $[0,1] \ldots \ldots \ldots \ldots \ldots \ldots \ldots \ldots \ldots \ldots \ldots . \ldots . \ldots . \ldots . \ldots 25$

M. S. Robertson, Cesàro partial sums of harmonic series expansions...... 829

John L. Selfridge and Ernst Gabor Straus, On the determination of numbers by their sums of a fixed order ........................ 847

Annette Sinclair, A general solution for a class of approximation

problems .................................

George Szekeres and Amnon Jakimovski, $(C, \infty)$ and $(H, \infty)$ methods of summation...................................... 867

Hale Trotter, Approximation of semi-groups of operators. ............. 887

L. E. Ward, A fixed point theorem for multi-valued functions ........... 921

Roy Edwin Wild, On the number of lattice points in $x^{t}+y^{t}=n^{t / 2} \ldots \ldots .929$ 